\section{Commentary: Trisomies and tribulations: Don't get blindsided by pulmonary complications after pediatric heart surgery}

Douglas M. Overbey, MD, Joseph W. Turek, $\mathrm{MD}, \mathrm{PhD}$, and Nicholas D. Andersen, MD

Operative decision making for children with trisomy 13 or 18 (T13/18) and congenital heart defects is a complex ethical calculus that must balance the increased risk of postoperative morbidity and mortality against the predicted increase in the quality or quantity of life in children with multisystem birth defects and limited life expectancy. Although surgery is being performed in an increasing number of T13/18 patients, it is important for families and physicians to understand the expected postoperative recovery and major complications that can occur following these procedures. Swanson and Colleagues ${ }^{1}$ present a retrospective case-control study of T13/18 patients who underwent cardiac operations over a recent 20 -year period of time at a single high-volume center. Of 14 case patients, all underwent a variation of ventricular septal defect repair and were matched 3:1 with controls with similar diagnoses and age. The principle aim of the study was to describe the prevalence of airway anomalies and pulmonary/airway complications following surgery.

Preoperative characteristics showed an increased incidence of preoperative respiratory support $(71 \%$ vs $14 \%$; $P<.001)$ and an increased incidence of critical airway designation $(50 \%$ vs $5 \% ; P<.001)$ in patients with T13/18. Postoperatively, there was a higher incidence of prolonged mechanical ventilation $(50 \%$ vs $7 \% ; P=.001)$, higher

\footnotetext{
From Division of Cardiovascular and Thoracic Surgery, Duke Children's Pediatric \& Congenital Heart Center, Duke Children's Hospital, Durham, NC.

Disclosures: The authors reported no conflicts of interest.

The Journal policy requires editors and reviewers to disclose conflicts of interest and to decline handling or reviewing manuscripts for which they may have a conflict of interest. The editors and reviewers of this article have no conflicts of interest.

Received for publication Sept 13, 2020; revisions received Sept 13, 2020; accepted for publication Sept 14, 2020; available ahead of print Sept 18, 2020.

Address for reprints: Nicholas D. Andersen, MD, Division of Cardiovascular and Thoracic Surgery, Duke University Medical Center, 2301 Erwin Rd, DUMC 3474, Durham, NC 27710 (E-mail: Nicholas.Andersen@Duke.edu).

J Thorac Cardiovasc Surg 2021;162:250-1

$0022-5223 / \$ 36.00$

Copyright (c) 2020 by The American Association for Thoracic Surgery

https://doi.org/10.1016/j.jtcvs.2020.09.052
}

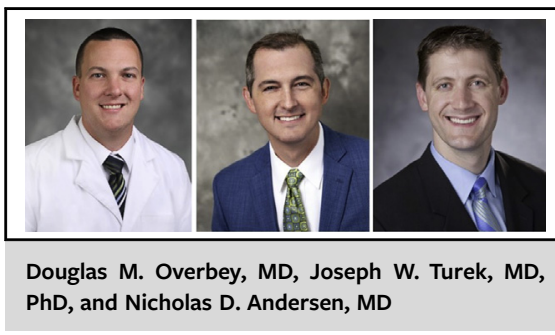

\begin{abstract}
CENTRAL MESSAGE
Pulmonary complications are ubiquitous following heart surgery in children with trisomy 13 or 18. These data may inform patient selection, family counseling, and perioperative airway management.
\end{abstract}

rate of reintubation $(50 \%$ vs $12 \% ; P=.01)$, higher rate of tracheitis or pneumonia $(43 \%$ vs $0 \% ; P<.001)$, longer duration of mechanical ventilation (7.5 vs 2 days; $P<.001$ ), and an overall longer length of stay (31 vs 8 days; $P<.001)$ in T13/18 patients. There were signals toward higher rates of tracheostomy and mortality in T13/18 patients that did not reach statistical significance. Most strikingly, postoperative pulmonary complications were almost uniformly experienced, affecting 13 of 14 (93\%) T13/18 patients. Further, $100 \%$ ( 7 of 7 ) of T13/18 patients who underwent a postoperative airway evaluation had significant findings. ${ }^{1}$

Although long-term T13/18 survival is poor, cardiac surgery can be performed in select patients with acceptable short-term survival. ${ }^{2}$ In the present cohort, $93 \%$ of $\mathrm{T} 13 /$ 18 patients survived to discharge, $70 \%$ survived to 1 year, and the median survival was 3.4 years.

The strength of this study is the detailed and granular description of the preoperative workup and postoperative course with specific details and durations of respiratory and infectious complications. This level of detail is not available through typical database studies. Limitations include the retrospective study design, small sample size, and a relatively long (20 years) accrual time, during which many aspects of operative and postoperative care have changed.

Overall, this study provides objective data to assist in counseling families before congenital heart surgery in T13/18 patients, and to establish reasonable expectations regarding postoperative recovery and potential long-term complications of surgery, including tracheostomy and 
ventilator dependence. Preoperative airway evaluation is essential, and the authors advocate for a multidisciplinary team approach, including consultation with palliative care. Postoperative pulmonary complications should be monitored closely along with a higher expectancy for reintubation or tracheostomy before discharge. At our center, we currently require a preoperative airway evaluation and assent for postoperative tracheostomy (in the event it is needed) before embarking on heart surgery in children with T13/18. This study appears to support these practices.

\section{References}

1. Swanson SK, Schumacher KR, Ohye RG, Zampi JD. Impact of trisomy 13 and 18 on airway anomalies and pulmonary complications after cardiac surgery. J Thorac Cardiovasc Surg. 2021;162:241-9.

2. Cooper DS, Riggs KW, Zafar F, Jacobs JP, Hill KD, Pasquali SK, et al. Cardiac surgery in patients with trisomy 13 and 18: an analysis of the Society of Thoracic Surgeons congenital heart surgery database. J Am Heart Assoc. 2019;8:e012349.
See Article page 241 .

\section{Commentary: Cardiac surgery in children with trisomy 13 and trisomy 18: "Is it the quality of life or the quantity?"}

Minoo N. Kavarana, MD

It is now well established that children with trisomy 13 and 18 have a very short life expectancy, with a median survival of less than 2 weeks. ${ }^{1}$ However, for those children who survive past 6 months, $50 \%$ to $60 \%$ may survive 10 years. ${ }^{1}$ In translocations that result in partial trisomy or mosaics, improved survival up to 27 years has been reported. ${ }^{2}$ Some parents choose to terminate pregnancy, whereas others proceed to make decisions based on their beliefs, understanding, and physician recommendations. Physicians too are often divided regarding treatment of these children, as some feel that aggressive treatments are futile whereas others defer to the parents' wishes.

Recent advances in surgical technique and postoperative intensive care unit (ICU) management have resulted in

\footnotetext{
$\overline{\text { From the Section }}$ of Pediatric Cardiothoracic Surgery, Medical University of South Carolina, Charleston, SC.

Disclosures: The author reported no conflicts of interest.

The Journal policy requires editors and reviewers to disclose conflicts of interest and to decline handling or reviewing manuscripts for which they may have a conflict of interest. The editors and reviewers of this article have no conflicts of interest.

Received for publication Sept 5, 2020; revisions received Sept 5, 2020; accepted for publication Sept 8, 2020; available ahead of print Sept 15, 2020.

Address for reprints: Minoo N. Kavarana, MD, FACS, Section of Pediatric Cardiothoracic Surgery, Pediatric Heart Transplantation and Mechanical Support, 10 McClennan Banks Dr, SJ 2190 N/MSC 918, Charleston, SC 29425 (E-mail: kavarana@musc.edu)

J Thorac Cardiovasc Surg 2021;162:251-2

$0022-5223 / \$ 36.00$

Copyright (c) 2020 by The American Association for Thoracic Surgery

https://doi.org/10.1016/j.jtcvs.2020.09.027
}

\section{Check for updates}

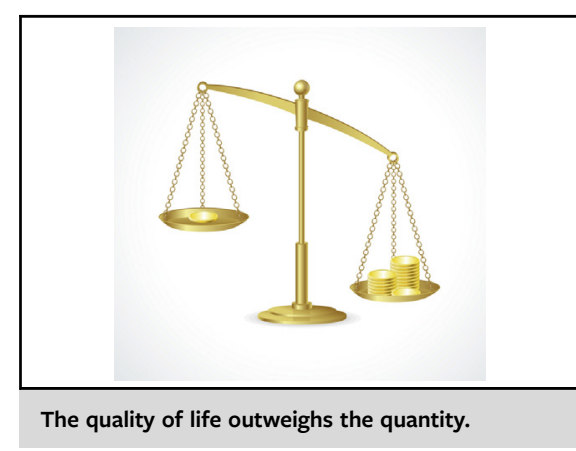

CENTRAL MESSAGE

Children with trisomy 13 and trisomy 18 with congenital heart disease can undergo corrective surgical repair with reasonable hospital survival but questionable medium-term survival.

significantly improved hospital mortality. ${ }^{3}$ Centers have therefore extended cardiac surgery to greater-risk populations with genetic syndromes including trisomy 13 and $18 .^{3}$ A study conducted by the Pediatric Cardiac Care Consortium found that patients who required prolonged preoperative ventilator support had worse outcomes. ${ }^{4}$ For patients who were ventilator dependent for more than 2 days preoperatively, only $33 \%$ were extubated before discharge, and $11 \%$ died. They demonstrated a $91 \%$ survival and concluded that most patients with trisomy 13 and 18 can survive cardiac surgery. ${ }^{4}$ A study from the Society of Thoracic Surgeons database confirmed that the hospital mortality was reasonable except for children who required preoperative mechanical ventilation, who had an 8 times greater hospital mortality. ${ }^{5}$ 